\title{
Upregulation of TCTP expression in human skin squamous cell carcinoma increases tumor cell viability through anti-apoptotic action of the protein
}

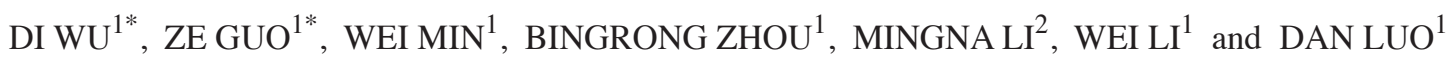 \\ Departments of ${ }^{1}$ Dermatology, and ${ }^{2}$ Pathology, The First Affiliated Hospital \\ of Nanjing Medical University, Nanjing 210029, P.R. China
}

Received September 26, 2011; Accepted December 21, 2011

DOI: $10.3892 /$ etm.2011.439

\begin{abstract}
The translationally controlled tumor protein (TCTP) is an anti-apoptotic protein, which is highly expressed in several human cancer types. However, the role of TCTP in skin cancers, including squamous cell carcinoma (SCC), has not been investigated. In this study, we analyzed the expression of TCTP in cutaneous SCC samples using immunohistochemistry in two epidermoid SCC cell lines, A431 and SCL-1, using western blot analysis. We further investigated the role of TCTP in skin cancinogenesis by silencing the TPT1 gene using small interfering RNA (siRNA) in the SCC cell line A431. Our results demonstrated that TCTP was overexpressed in cutaneous SCC cells, compared to normal skin keratinocytes. In addition, the expression of TCTP in skin SCC significantly increased with the grade of malignancy. Western blot analysis further confirmed that the expression of TCTP in the cell lines, A431 and SCL-1, was significantly higher compared to that in the normal keratinocyte cell line, HaCaT. The expression of TCTP in A431 cells was significantly downregulated by transfection with our specifically designed TCTP siRNA. We found that downregulation of TCTP expression was associated with decreased cell proliferation and increased apoptosis in A431 cells. These results suggest that the TPT1 gene may be a potential therapeutic target in skin SCC through a siRNA approach.
\end{abstract}

\section{Introduction}

Human cutaneous squamous cell carcinoma (SCC) is the sixth most common cancer in the world. It arises in the keratinocytes

Correspondence to: Professor Dan Luo, Department of Dermatology, The First Affiliated Hospital of Nanjing Medical University, Nanjing 210029, P.R. China

E-mail: daniluo2005@yahoo.com.cn

*Contributed equally

Key words: translationally controlled tumor protein, squamous cell carcinoma, gene silencing of the epidermis or its appendages. Skin SCC may arise de novo or develop from precursor lesions, including actinic keratosis (AK) or Bowen's disease. The majority of primary skin SCCs have a good prognosis and are usually curable in comparison with other cancer types; however, the potential for invasion and metastases significantly contributes to mortality. Based on the degree of tumor differentiation, SCC can be graded into three histological categories; well-differentiated, moderatelydifferentiated and poorly-differentiated. Poorly-differentiated SCCs usually exhibit a higher risk of recurrence and metastasis. However, biological behaviors can be difficult to predict from the status of differentiation of the tumor cells. Therefore, a novel molecular biomarker that reveals associations between the aggressive, invasive and metastatic behaviors of cutaneous SCC needs to be identified.

The translationally controlled tumor protein (TCTP), a 172-amino acid anti-apoptotic polypeptide, was originally identified in the Ehrlich ascites tumor cell line (1). It is a cell growth-associated protein that is ubiquitously present in a wide variety of organisms and is pivotal in the development of various organisms (2-4). It is present extra- and intracellularly and has been implicated in a number of cellular functions related to cell growth and apoptosis (5). The TCTP gene is significantly downregulated in revertant tumor cells, and it was therefore suggested that inhibiting the expression of TCTP could revert cancer cells back into normal phenotypes (6). Certain stimuli, including dioxin, heavy metals, growth factors and vitamin $\mathrm{D}$, can regulate the expression of TCTP (7-9). TCTP also interacts with numerous cellular proteins, including tubulin (6), translation elongation factor IA (eEF1A) and associated guanine nucleotide exchange factor (eEF1B-b) (10), Mcl-1 (11), TSAP6 (12), Na,K-ATPase (13), and Bcl-XL (14). Recently, the anti-apoptotic activity of TCTP has been reported, which could be related to its interaction with Mcl-1 and/or Bcl-XL (11,15). TCTP is highly expressed in several cancer types; however, the expression of TCTP has not been investigated in cutaneous SCC. Its roles in skin carcinogenesis also need to be elucidated.

In this study, we investigated the expression of TCTP in cutaneous SCC and its function in skin carcinogenesis by using a small interfering RNA (siRNA) gene silencing approach. 


\section{Materials and methods}

Specimens. From a total of 65 cases of cutaneous SCC, paraffin-embedded samples were retrieved from the Department of Pathology at The First Affiliated Hospital of Nanjing Medical University, China. All hematoxylin and eosin-stained slides diagnosed as SCC were reviewed by two independent pathologists using the accepted histopathological criteria. Five healthy individuals without skin disease were used as controls.

The histological grading of the SCC was reevaluated for this study according to the following classification; well-differentiated, moderately-differentiated and poorly-differentiated SCCs, which were recognized as grade I, II and III, respectively. There were 24 cases of grade I, 23 cases of grade II and 18 cases of grade III. Of the total 65 SCC cases, nine patients had metastatic SCC.

Immunohistochemical analysis. Paraffin-embedded blocks were resected as $5-\mu \mathrm{m}$ sections and mounted onto positively charged Superfrost slides. One section from each sample was stained with hematoxylin and eosin to facilitate histological assessment. The immunostaining was performed according to a standard protocol. Briefly, paraffin sections from the SCC specimens and normal patients were deparaffinized in xylene and rehydrated through a graded series of ethanol. Following blocking samples with 3\% hydrogen peroxide for $10 \mathrm{~min}$, the samples were subjected to microwave antigen retrieval using in $0.01 \mathrm{M}$ citrate buffer for $15 \mathrm{~min}$. The slides were then washed twice with PBS buffer and incubated with primary polyclonal anti-TCTP antibody (1:100 dilutions; bs-3578R; Beijing Biosynthesis Biotechnology Co. Ltd, Beijing, China) at room temperature for $30 \mathrm{~min}$. Then the sections were incubated with goat anti-mouse horseradish peroxidase (HRP)-streptavidin immunoglobulin (Abcam, Cambridge, UK) at room temperature for $30 \mathrm{~min}$.

Following counterstaining the slides with hematoxylin and mounting on cover slips, the slides were analyzed by Image Pro Plus 6.0 (Media Cybernetics, Silver Spring, MD, USA) for measurement of the optical density. Any evidence of cytoplasmic or membrane staining for TCTP was considered as positive. The immunostaining results were evaluated by defining a threshold of positive staining for all sections prior to automated processing. The intensity was averaged from ten fields of view. All images analyzed with IPP 6.0 were counter-checked by an experienced histopathologist.

Cell culture. The epidermoid carcinoma cell lines, A431 and SCL-1, and the keratinocyte cell line, HaCaT, were gifts kindly provided by $\mathrm{Dr} \mathrm{Gu}$ at the Department of Dermatology of Changhai Hospital, Shanghai, China. Cells were cultured in either $25 \mathrm{~cm}^{2}$ tissue culture flasks, 6- or 96-well plates or tissue culture glass slides at $37^{\circ} \mathrm{C}$ in a $5 \% \mathrm{CO}_{2}$ environment. DMEM medium with $10 \%$ fetal bovine serum (FBS) was used for cell growth.

Western blot analysis. Protein extracts were prepared from the cell lines using lysis buffer containing $50 \mathrm{mM}$ Tris- $\mathrm{HCl}(\mathrm{pH} 7.4)$, $1 \%$ NP-40 (Sigma, St. Louis, MO, USA), $0.25 \%$ sodium deoxycholate, $150 \mathrm{mM} \mathrm{NaCl}, 1 \mathrm{mM}$ phenylmethylsulfonyl fluoride, $1 \mu \mathrm{g} / \mathrm{ml}$ aprotinin, $1 \mu \mathrm{g} / \mathrm{ml}$ leupeptin and $1 \mu \mathrm{g} / \mathrm{ml}$ pepstatin.
The protein concentrations were determined using the Bradford assay. The protein lysate $(50 \mu \mathrm{g})$ was separated on a $10 \%$ SDS-PAGE gel and transferred onto polyvinylidene difluoride membranes (Millipore, Billerica, MA, USA) for western blot analysis detection. The blot was blocked with 5\% non-fat dry milk in a buffer containing $10 \mathrm{mM}$ Tris (pH 7.5), $100 \mathrm{mM}$ $\mathrm{NaCl}$ and $0.1 \%$ Tween-20 (Sigma). The blot was washed and incubated with primary polyclonal anti-TCTP antibody (1:1000 dilution) for $1 \mathrm{~h}$ and then incubated for $30 \mathrm{~min}$ with secondary goat anti-rabbit antibody conjugated with horseradish peroxidase (1:3000 dilution). Immunoreactive protein signals were visualized by an enhanced chemiluminescence kit (Thermo Scientific, Amersham, USA).

siRNA treatment. The TCTP protein is encoded by the TPTI gene. The siRNAs for the TPT1 gene were 21 base pairs of oligonucletides synthesized at Biomics Biotechnologies Company Ltd. (Nantong, China). Three specific siRNAs were designed to start at various nucleotide sites on the TPTI gene cDNA: TPT1-272-siRNA (sense siRNA, 5'-GGUACCG AAAGCACAGUAAdTdT-3'; antisense siRNA, 5'-UUACUGU GCUUUCGGUACCdTdT-3'); TPT1-316-siRNA (sense siRNA, 5'-CCAUCACCUGCAGGAAACAdTdT-3'; antisense siRNA， 5'-UGUUUCCUGCAGGUGAUGGdTdT-3'); TPT1-351-siRNA (sense siRNA, 5'-ACCAUCACCUGCAGG AAACdTdT-3'; antisense siRNA, 5'-GUUUCCUGCAGG UGAUGGUdTdT-3'). A negative control siRNA (siRNA-NC) that shared a poor homology with the human genome sequence was designed as the negative control (sense siRNA, 5'-CCGA ACGUGUCACGUUCdTdT-3'; antisense siRNA, 5'-GAACG UGACACGUUCGGdTdT-3'). We also designed a FAM-labeled siRNA-NC probe to examine the transfection efficiency.

All procedures were performed in an RNAse-free environment using RNAse-free water. Cells were then transfected with an approximate final concentration of $100 \mathrm{nM}$ of siRNA duplexes using Lipofectamine (Invitrogen Life Technologies, Carlsbad, CA, USA). Cells were collected at 24, 48 and $72 \mathrm{~h}$ following the transfection and used for cell viability, fluorescence microscopy studies and RT-PCR analysis.

RT-PCR analysis. mRNA was isolated from mock-transfected and TPTI-siRNA transfected A431 cells using TRIzol reagent according to the manufacturer's instructions (Invitrogen). The mRNA wasthenconverted tocDNAusing aRT-PCRkit(Ambion, Kyoto, Japan). Expression of TPTI in the cDNA samples was determined using PCR using gene-specific TPT1 primers (sense primer 5'-TCTATCACCTGTCATCATAACTG-3'; antisense primer 5'-CCACTCCAAATAAATCACAGTC-3'), which were designed by Biomics Biotechnologies. PCR parameters were as follows: $30 \mathrm{sec}$ of denaturation at $95^{\circ} \mathrm{C}$, $30 \mathrm{sec}$ of primer annealing at $55^{\circ} \mathrm{C}$ and $20 \mathrm{sec}$ of primer extension at $72^{\circ} \mathrm{C}$; the cycle was repeated 40 times. A final extension of $5 \mathrm{~min}$ at $72^{\circ} \mathrm{C}$ was performed prior to storing the samples at $4^{\circ} \mathrm{C}$. Following PCR, the products were analyzed by $1 \%$ agarose gel electrophoresis.

Cell viability assay. A431 cells $\left(2 \times 10^{3}\right.$ cells $\left./ \mathrm{ml}\right)$ were cultured in 96-well plates until they reached 50\% confluency. Cells were then transfected with a final concentration of $100 \mathrm{nM}$ TCTP siRNA. A total of $72 \mathrm{~h}$ following siRNA transfec- 
tion, cell viability was determined by a Cell Counting kit- 8 (CCK-8; Beyotime Institute of Biotechnology, Jiangsu, China). Briefly, $10 \mu \mathrm{l}$ of water-soluble formazan dye was added to the mock-transfected and siRNA-transfected cells, and plates were incubated for $2 \mathrm{~h}$ in $5 \% \mathrm{CO}_{2}$. The developed color was measured at $450 \mathrm{~nm}$ using an ELISA plate reader.

Hoechst staining. A431 cells were cultured in 6-well tissue culture plates until they were transfected with TCTP siRNA. Following 72-h incubation, the cells were stained with Annexin V-FITC and propidium iodide. Stained cells were visualized with a fluorescence microscope (Nikon Eclipse ET2000-E, Japan).

Cell apoptosis detection. A431 cells were harvested by trypsinization $72 \mathrm{~h}$ following transfection and centrifugation $\mathrm{x} 1000 \mathrm{~g}$ for $5 \mathrm{~min}$. Cells were fixed with $70 \%$ ethanol overnight at $4^{\circ} \mathrm{C}$. Following a wash in PBS, the cells were incubated with $1 \mathrm{mg} / \mathrm{ml} \mathrm{RNase}$ A (Sigma) for $30 \mathrm{~min}$ at $37^{\circ} \mathrm{C}$ and then with $100 \mathrm{ug} / \mathrm{ml}$ propidium iodide (Sigma) for $20 \mathrm{~min}$ at $4^{\circ} \mathrm{C}$. The apoptosis of cells was evaluated by flow cytometry on a FACSort flow cytometer (BD Biosciences FACSCalibur). A total of 20,000 events were determined by the forward side scatter (FSC) signal to exclude cell aggregates. The data were analyzed using the CellQuest software.

Statistical analysis. Statistical analysis was performed using SPSS $^{\circledR}$ for Windows version 16.0 (SPSS, Chicago, IL, USA). The statistical analysis for the TCTP immunostaining in different groups was performed using a nonpaired t-test.

\section{Results and Discussion}

Although TCTP appears to be ubiquitous and multifunctional, its expression and role in cutaneous SCC have not been investigated. In this study, we first examined TCTP expression in skin SCCs using immunohistochemistry. Then we further investigated its functions in epidermoid carcinoma cell lines using a siRNA gene silencing approach.

Five normal cases exhibited slight immunostaining of TCTP in skin keratinocytes majorly located in the basal layers (Fig. 1A). However, all skin SCC samples displayed typical diffused cytoplasmic staining of TCTP. More importantly, the density of immunostaining was significantly increased with the grade of malignancy. Well-differentiated SCCs (grade I) exhibited relatively weak cytoplasmic staining of the tumor cells, with an average optical density (OD) of $0.155 \pm 0.061$, but a horny pearl was evident as a typical feature (Fig. 1B). Moderately-differentiated SCCs (grade II) were observed with moderate cytoplasmic staining, with an average OD of $0.255 \pm 0.031$ (Fig. 1C). Poorly-differentiated SCC (grade III) displayed robust cytoplasmic staining with an average OD of $0.341 \pm 0.135$ (Fig. 1D). The expression of the TCTP protein revealed a statistically significant difference between grade II and grade I SCCs $(\mathrm{p}<0.01)$. The expression of TCTP in grade III SCCs was also significantly higher than in grade I and II SCC samples ( $\mathrm{p}<0.01 ; \mathrm{p}<0.05$, respectively). TCTP was initially reported to be a cytoplasmic protein (16). However, other studies later reported the nuclear localization of TCTP by immunohistochemical analysis (11). Based on our results that revealed TCTP is a cytoplasmic protein in skin SCC tumor cells, we predict that TCTP may shuttle between the cytoplasm and the nucleus, depending on the cell cycle phase, differentiation state or environmental conditions. We presume that upregulation of TCTP in skin SCC may contribute to tumor growth through the promotion of cell growth and inhibition of apoptosis in tumor cells, and eventually result in increasing metastasis. In our study, among the 65 cases of skin SCCs, there were nine patients with lymph node metastatic SCC. However, there is no statistically significant difference in TCTP expression between the secondary and primary cutaneous SCCs (OD: 0.290 \pm 0.201 vs. 0.236 $\pm 0.110, \mathrm{p}>0.05$ ). Thus, the expression of TCTP appeared to be not significantly associated with lymph node metastasis in cutaneous SCC, which was consistent with an existing study (17).

TCTP has been found to be expressed in several healthy and tumor cells, including erythrocytes, hepatocytes, macrophages, platelets, keratinocytes, erythroleukemia cells, gliomas, melanomas, hepatoblastomas and lymphomas, but it is not detected in renal cell carcinoma (16). Using immunohistochemical analysis, our study indicates that the expression of TCTP is upregulated in skin SCCs. More importantly, the level of TCTP expression is significantly associated with the grade of cutaneous SCCs. The poorly-differentiated SCC had a higher level of TCTP expression. Next, using western blot analysis, we further demonstrated that TCTP had a higher expression level in the two epidermoid SCC cell lines in comparison with normal keratinocytes. Western blot analysis results revealed that the skin SCC cell lines, A431 and SCL-1, expressed fold higher levels of TCTP than the normal keratinocyte cell line, HaCaT (Fig. 1E). Between these two SCC cell lines, A431 cells express slightly higher TCTP than SCL-1 cells (Fig. 1E). Therefore, we selected the SCC cell line, A431, to further investigate the role of TCTP in skin SCC using RNA interference.

Previously, siRNA techniques that efficiently block specific target gene expression have been demonstrated. Thus, we selected this method to observe the effect of TCTP in A431 SCC cells when TCTP expression was downregulated by specific siRNAs. For this purpose, we designed three TPT1-siRNAs from various nucleotide sites initiating from the start code in the TPT1 cDNA, which were named TPT1-272-siRNA, TPT1-316-siRNA and TPT1-351-siRNA. Since the tumor cells with the highest level of endogenous protein are likely to get the most prominent inhibitory effect from the antisense oligonucleotides (18), we selected the A431 cell line with the highest level of TCTP expression for the siRNA gene silencing investigation. A431 cells were first transfected with siRNA-NC to examine the transfection efficiency. Varying siRNA concentrations and siRNA transfection reagents were compared by observing the levels of green fluorescence to obtain the optimal transfection efficiency. The highest transfection efficiency reached $98 \%$ (Fig. 2B and C). Hoechst staining was used to confirm the location of the cells (Fig. 2A and C). Cells were then transfected with three types of TPT1-siRNAs: TPT1-272-siRNA, TPT1-316-siRNA and TPT1-351-siRNA. The silencing of the target gene was confirmed by RT-PCR. Time course analyses revealed that $72 \mathrm{~h}$ following transfection with TPT1-351-siRNA, there was a significant decrease in the level of TCTP mRNA transcripts in the transfected cells, 

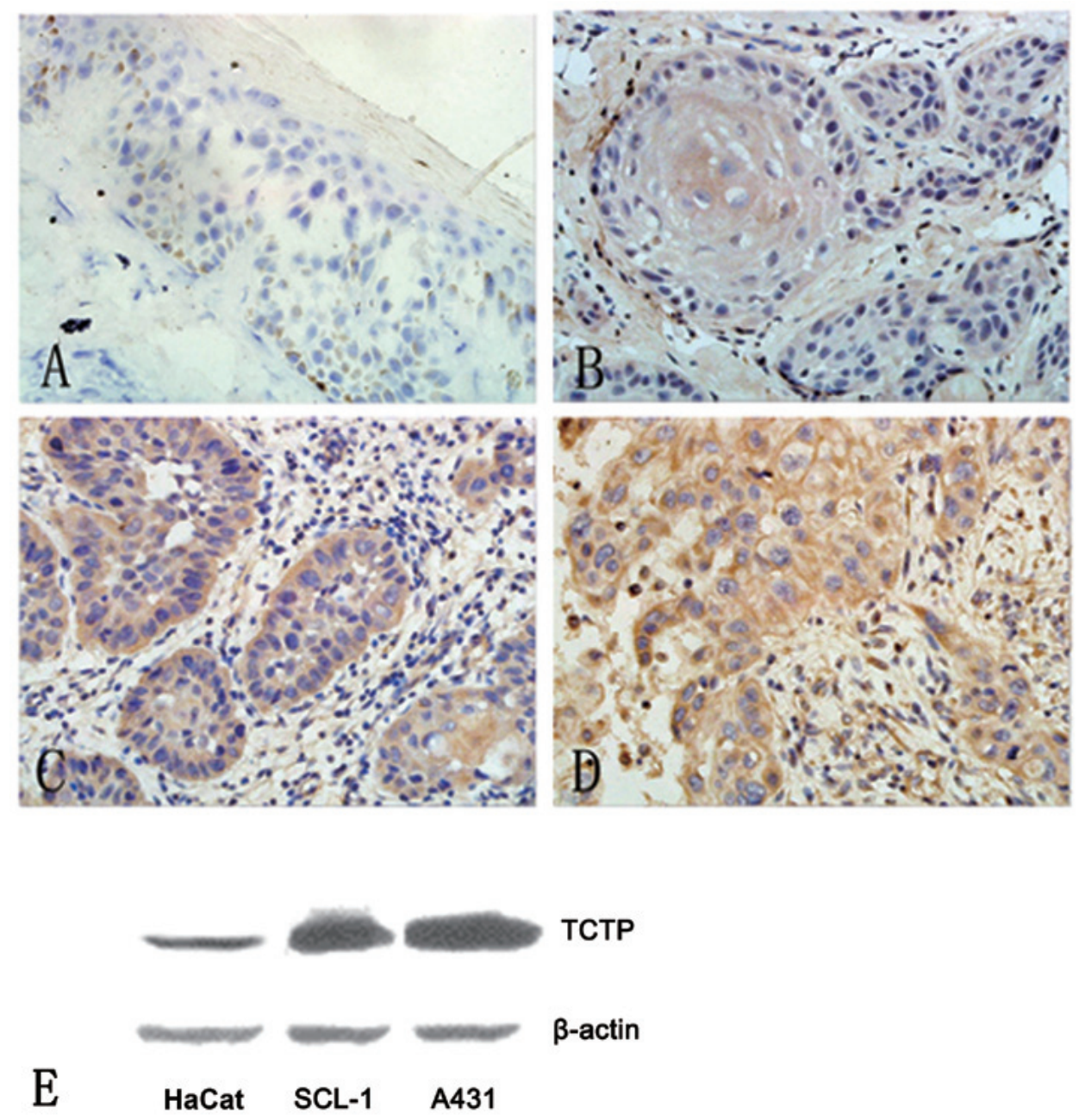

Figure 1. (A-D) Translationally controlled tumor protein (TCTP) expression in normal skin tissues and tumor specimens with cutaneous squamous cell carcinoma (SCC). (A) All normal cases exhibited slight staining of cells. (B) Well-differentiated SCCs (grade I) exhibited weak cytoplasmic staining of the tumor cells, with an average optical density (OD) of $0.155 \pm 0.061$. (C) Moderately-differentiated SCC (grade II) exhibited moderate cytoplasmic TCTP staining, with an average OD of $0.255 \pm 0.031$. (D) Poorly-differentiated SCC (grade III) exhibited very strong cytoplasmic staining for TCTP, with an average OD of $0.341 \pm 0.135$. Original magnification, $x 400$. (E) Western blot analysis of TCTP expression in keratinocyte cell line (HaCaT) and two SCC cell lines (A431 and SCL-1). $\beta$-actin served as the protein loading control.

whereas TPT1-272-siRNA and TPT1-316-siRNA induced a significant reduction in TCTP transcript, but a relatively lower reduction compared to TPT1-351-siRNA (Fig. 2D). These results indicate that TPT1-351-siRNA most effectively and specifically downregulated the expression of the TCTP protein in the A431 cell line. We therefore selected the TPT1-351-siRNA and the time of $72 \mathrm{~h}$ for the next function studies.

Flow cytometry was used to determine the anti-apoptotic effect of TCTP on the SCC cell line, A431. Transfection with TPT1-351-siRNA resulted in producing significantly more apoptotic cells in the SCC cell line A431 (Fig. 3A). The proportion of apoptotic cells out of the total A431 cells transfected with TPT1-351-siRNA was $14.69 \pm 1.03 \%$, whereas the proportion of apoptotic cells in the control group treated with Lipofectamine alone was $0.4 \pm 0.03 \%$. The percentage of apoptotic cells in the negative control group treated with siRNA-NC was $1.21 \pm 0.055 \%$ (Fig. 3B). Our results revealed that the A431 cells transfected with TPT1-351-siRNA underwent considerably more apoptosis compared to the cells transfected with control siRNA-NC or Lipofectamine alone (Fig. 3A and B).
Cells that were transfected with TPT1-351-siRNA for $72 \mathrm{~h}$ were further selected for the cell proliferation assay, which demonstrated a significant decrease in cell proliferation in the TPT1-351-siRNA-transfected A431 cells using the CCK-8 assay. These studies revealed that knocking down the TCTP gene resulted in decreased cell proliferation in the A431 cells compared to cells that were transfected with a control siRNA-NC or Lipofectamine alone ( $<<0.05$; Fig. $3 \mathrm{C}$ ).

$\mathrm{Li}$ et al were the first to demonstrate the anti-apoptotic property of TCTP. Another study then revealed similar results that altered levels of TCTP affect the cell proliferation and apoptotic states in hepatoma cells (11). Furthermore, a recent study has also demonstrated that TCTP acts as a negative regulator of apoptosis in lung cancer. TCTP prevents apoptosis by destabilizing p53, and the overexpression of TCTP promotes the degradation of p53 (19). In this study, we provided evidence to support the theory that overexpression of TCTP in skin SCCs leads to increased tumor cell proliferation through an anti-apoptotic function. Several anti-apoptotic mechanisms have been proposed for TCTP $(17,20)$. However, the molecular mechanisms concerning how TCTP acts as an anti-apoptotic 

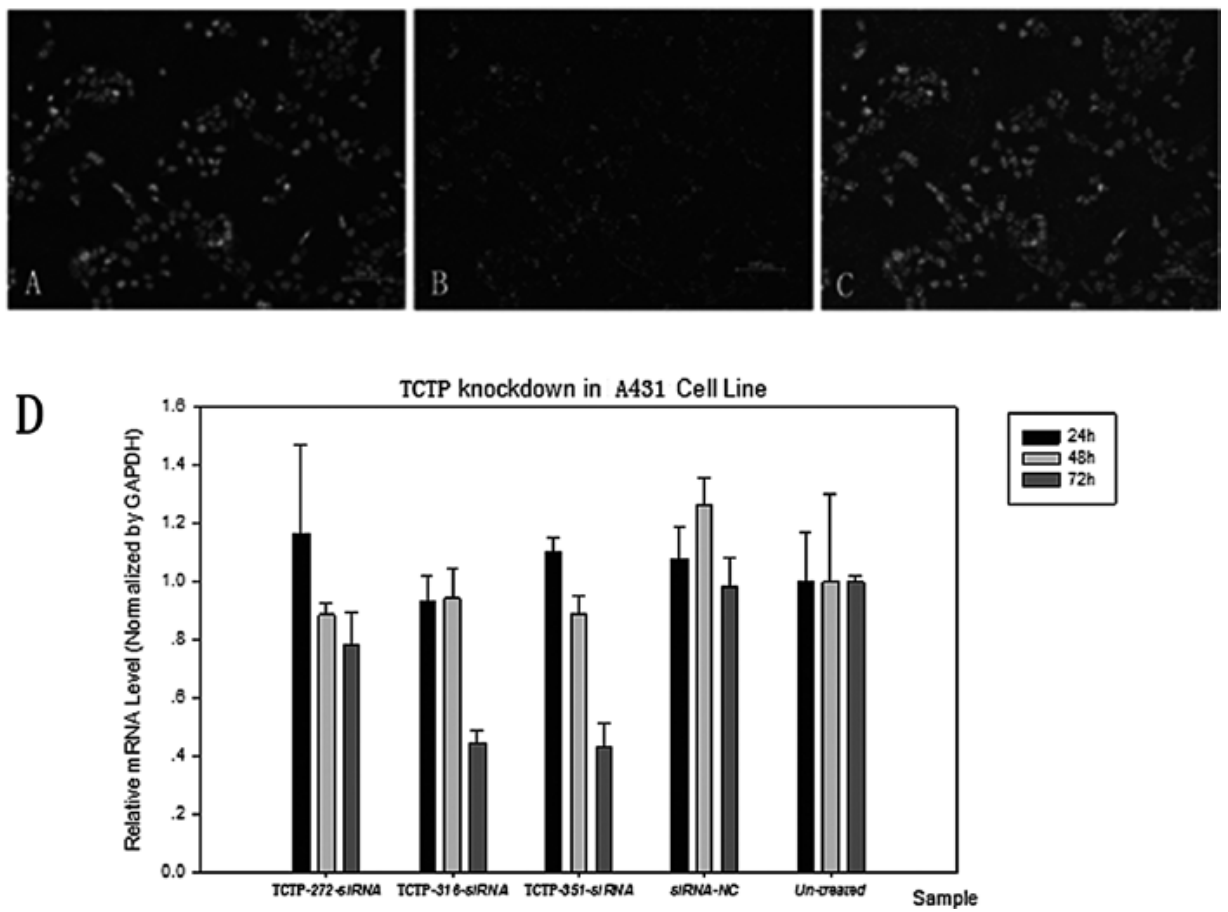

Figure 2. (A-C) Hoechst staining and FAM green fluorescence were used to detect the transfection efficiency. (A) Hoechst staining of cells $72 \mathrm{~h}$ following the transfection of A431 cells. (B) The green fluorescence of cells $72 \mathrm{~h}$ following the transfection of A431 cells. (C) The merge of the two types of fluorescence. The scale bar is $100 \mu \mathrm{m}$. (D) Gene silencing of the TCTP gene by siRNA transfection. A431 cells $\left(10^{4}\right.$ cells $\left./ \mathrm{ml}\right)$ were transfected with TPT1-272-siRNA, TPT1-316-siRNA, TPT1-351-siRNA or siRNA-NC Lipofectamine reagent only. A431 cells transfected with Lipofectamine only served as untreated control. mRNAs extracted from cells at 24,48 and $72 \mathrm{~h}$ following the transfection were used to detect the expression of the TPT1 gene by RT-PCR. Results revealed that the expression of the TPT1 gene was significantly silenced by siRNA transfection; TPT1-351-siRNA had the best knockdown effect $72 \mathrm{~h}$ following transfection. TCTP, translationally controlled tumor protein; siRNA, small interfering RNA.

A

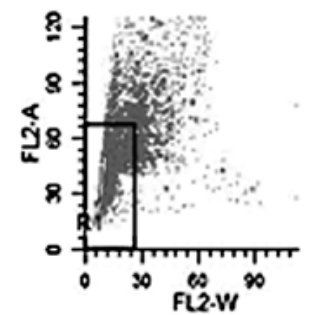

Negativo

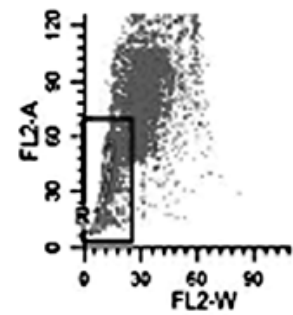

Control

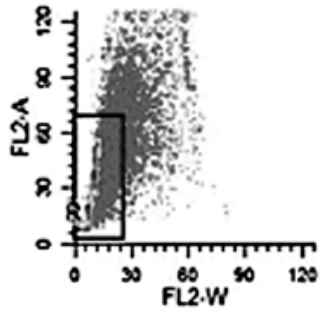

SIRNA
B

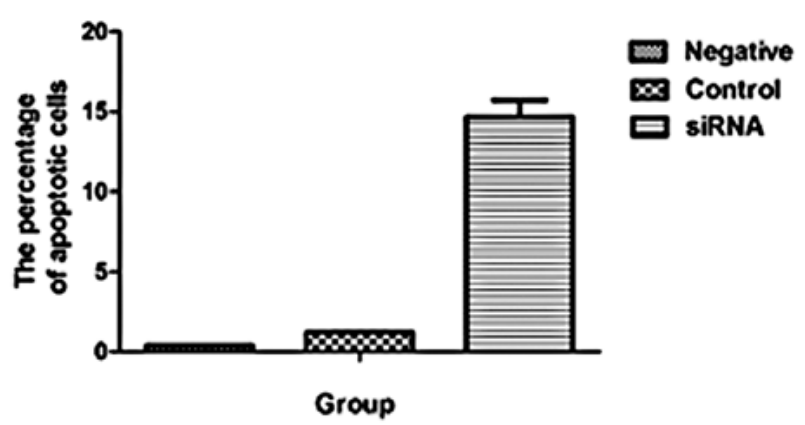

C

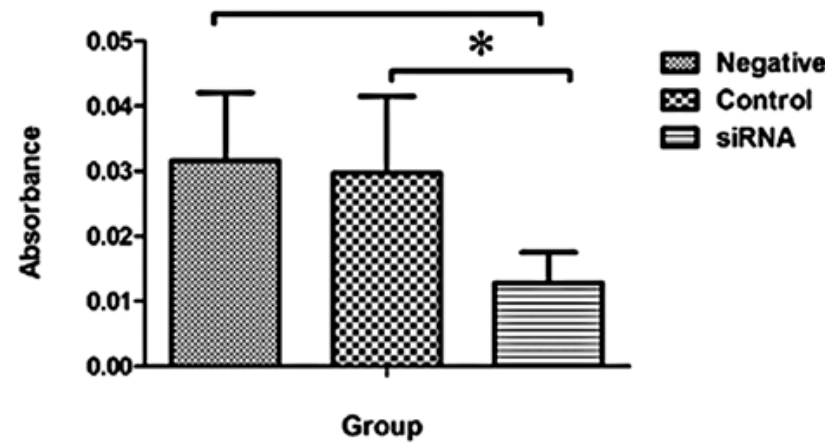

Figure 3. (A and B) Flow cytometer detection of apoptosis in the squamous cell carcinoma (SCC) cell line, A431. Cells (104 cells $/ \mathrm{ml})$ were transfected with Lipofectamine reagent only (negative group), siRNA-NC (control group) or TPT1-351-siRNA (siRNA group) for $72 \mathrm{~h}$. Then the cells were fixed with $70 \%$ ethanol and analyzed by flow cytometry. The presented results are representative of one of three similar experiments. (C) TPT1-351-siRNA transfection inhibits the growth of A431 cells. Cells $\left(2 \times 10^{3} \mathrm{~A} 431 \mathrm{cells} / \mathrm{ml}\right)$ were cultured in 96-well tissue culture plates and transfected with TPT1-351-siRNA (siRNA group) or siRNA-NC (control group). The viability of siRNA and control group cells at various time-points was analyzed by adding $10 \mu l$ formazan dye. The intensity of the color was measured by an ELISA plate reader at an absorbance spectrum of $450 \mathrm{~nm}$.There is a statistically significant difference between the siRNA and the control group ( $\mathrm{P}<0.05)$. siRNA, small interfering RNA; TCTP, translationally controlled tumor protein. 
protein in skin tumors, including SCC, need to be further elucidated. Additional studies are in progress in our laboratory to elucidate the anti-apoptotic mechanisms of TCTP, as well as the siRNA delivery route and its therapeutic potential against $\mathrm{SCC}$ in animal models.

In conclusion, TCTP is highly expressed in human skin squamous cell carcinoma. The level of TCTP expression increases with the grade of cutaneous SCCs. Downregulation of TCTP in the SCC cell line by a siRNA gene silencing approach leads to decreased cell viability and increased apoptosis. Our findings indicate that TCTP could be a candidate gene for the development of diagnostic and therapeutic strategies for skin SCC.

\section{Acknowledgements}

The study was supported by the National Natural Science Foundation of China (grant no. 30771946 and grant no. 81000700).

\section{References}

1. Bohm H, Benndorf R, Gaestel M, Gross B, Nurnberg P, Kraft R, Otto A and Bielka H: The growth-related protein P23 of the Ehrlich ascites tumor: translational control, cloning and primary structure. Biochem Int 19: 277-286, 1989.

2. Bonnet C, Perret E, Dumont X, Picard A, Caput D and Lenaers G: Identification and transcription control of fission yeast genes repressed by an ammonium starvation growth arrest. Yeast 16: 23-33, 2000.

3. MacDonald SM, Rafnar T, Langdon J and Lichtenstein LM: Molecular identification of an IgE-dependent histamine-releasing factor. Science 269: 688-690, 1995.

4. Chen SH, Wu PS, Chou CH, Yan YT, Liu H, Weng SY, et al: A knockout mouse approach reveals that TCTP functions as an essential factor for cell proliferation and survival in a tissue- or cell type-specific manner. Mol Biol Cell 18: 2525-2532, 2007.

5. Telerman A and Amson R: The molecular programme of tumour reversion: the steps beyond malignant transformation. Nat Rev Cancer 9: 206-216, 2009.

6. Tuynder M, Susini L, Prieur S, Besse S, Fiucci G, Amson R and Telerman A: Biological models and genes of tumor reversion: cellular reprogramming through tpt1/TCTP and SIAH-1. Proc Natl Acad Sci USA 99: 14976-14981, 2002.

7. Oikawa K, Ohbayashi T, Mimura J, Fujii-Kuriyama Y, Teshima S, Rokutan K, Mukai K and Kuroda M: Dioxin stimulates synthesis and secretion of IgE-dependent histamine-releasing factor. Biochem Biophys Res Commun 290: 984-987, 2002.
8. Sturzenbaum SR, Kille P and Morgan AJ: Identification of heavy metal induced changes in the expression patterns of the translationally controlled tumour protein (TCTP) in the earthworm Lumbricus rubellus1. Biochim Biophys Acta 1398: 294-304, 1998.

9. Vercoutter-Edouart AS, Czeszak X, Crepin M, Lemoine J, Boilly B, Le Bourhis X, Peyrat JP and Hondermarck H: Proteomic detection of changes in protein synthesis induced by fibroblast growth factor-2 in MCF-7 human breast cancer cells. Exp Cell Res 262: 59-68, 2001.

10. Langdon JM, Vonakis BM and MacDonald SM: Identification of the interaction between the human recombinant histamine releasing factor/translationally controlled tumor protein and elongation factor-1 delta (also known as eElongation factor-1B beta). Biochim Biophys Acta 1688: 232-236, 2004.

11. Li F, Zhang D and Fujise K: Characterization of fortilin, a novel antiapoptotic protein. J Biol Chem 276: 47542-47549, 2001.

12. Amzallag N, Passer BJ, Allanic D, Segura E, Thery C, Goud B, Amson R and Telerman A: TSAP6 facilitates the secretion of translationally controlled tumor protein/histamine-releasing factor via a nonclassical pathway. J Biol Chem 279: 46104461012, 2004.

13. Jung J, Kim M, Kim MJ, Kim J, Moon J, Lim JS, Kim M and Lee K: Translationally controlled tumor protein interacts with the third cytoplasmic domain of Na,K-ATPase alpha subunit and inhibits the pump activity in HeLa cells. J Biol Chem 279: 49868-49875, 2004.

14. Yang Y, Yang F, Xiong Z, Yan Y, Wang X, Nishino M, et al: An $\mathrm{N}$-terminal region of translationally controlled tumor protein is required for its antiapoptotic activity. Oncogene 24: 4778-4788, 2005.

15. Liu H, Peng HW, Cheng YS, Yuan HS and Yang-Yen HF: Stabilization and enhancement of the antiapoptotic activity of mcl-1 by TCTP. Mol Cell Biol 25: 3117-3126, 2005.

16. Sanchez JC, Schaller D, Ravier F, Golaz O, Jaccoud S, Belet M, et al: Translationally controlled tumor protein: a protein identified in several nontumoral cells including erythrocytes. Electrophoresis 18: 150-155, 1997.

17. Susini L, Besse S, Duflaut D, Lespagnol A, Beekman C, Fiucci G, et al: TCTP protects from apoptotic cell death by antagonizing bax function. Cell Death Differ 15: 1211-1220, 2008.

18. Hu TH, Huang CC, Liu LF, Lin PR, Liu SY, Chang HW, et al: Expression of hepatoma-derived growth factor in hepatocellular carcinoma. Cancer 98: 1444-1456, 2003.

19. Rho SB, Lee JH, Park MS, Byun HJ, Kang S, Seo SS, et al: Anti-apoptotic protein TCTP controls the stability of the tumor suppressor p53. FEBS Lett 585: 29-35, 2011.

20. Graidist P, Yazawa M, Tonganunt M, Nakatomi A, Lin CC, Chang JY, et al: Fortilin binds $\mathrm{Ca}^{2+}$ and blocks $\mathrm{Ca}^{2+}$-dependent apoptosis in vivo. Biochem J 408: 181-191, 2007. 